\title{
Insecticides Currently Used on Vegetables ${ }^{1}$
}

\author{
S. E. Webb and P. A. Stansly ${ }^{2}$
}

The following table lists many of the common insecticides currently labeled for use on vegetables in Florida. A number of new materials have been registered in the past few years or have had additional crops added to their labels. Some older organophosphate insecticides are now restricted to just a few crops, a result of recent rulings related to the Food Quality Protection Act. Changes continue, thus this listing may not be totally accurate at the time of printing.

No attempt has been made to list all available formulations. Some are listed under "Signal Word," when different formulations differ in toxicity. Many of the listed insecticides are limited to specific vegetables. Specific crop recommendations and pesticide labels should be consulted for more detailed information.

Insects can become resistant to any insecticide if it is used repeatedly. This also applies to alternating insecticides with similar modes of action, for example following a soil application of Admire with foliar applications of Actara or Assail (all neonicotinoids, MOA 4). To complicate matters, some products, such as Voliam Flexi, come premixed with two different modes of action. In general, pesticides with the same mode of action should be used no more than twice in any crop cycle if residual activity is short, and only once if residual activity is long. It is important that successive generations of a pest not be exposed to the same pesticide mode of action. To aid in developing a spray program, we have included a column with a code number for the mode of action of each insecticide. A footnote lists the mode of action associated with the code. In addition to alternating insecticides with different modes of action, integrating other non-chemical control measures in a pest management program should help to delay resistance.

1. This document is ENY-419 (IG018), one of a series of the Entomology and Nematology Department, Florida Cooperative Extension Service, Institute of Food and Agricultural Sciences, University of Florida. First published June 2000. Revised April 2012. Please visit the EDIS Website at http://edis.ifas.ufl. edu.

2. S. E. Webb, associate professor/Extension entomologist, Entomology and Nematology Department, Cooperative Extension Service, Institute of Food and Agricultural Sciences, University of Florida, Gainesville, FL 32611, and P. A. Stansly, professor, Entomology and Nematology Department, Southwest Florida Research and Education Center, University of Florida, Immokalee, FL 34142.

The use of trade names in this publication is solely for the purpose of providing specific information. UF/IFAS does not guarantee or warranty the products named, and references to them in this publication do not signify our approval to the exclusion of other products of suitable composition. All chemicals should be used in accordance with directions on the manufacturer's label. Use pesticides safely. Read and follow directions on the manufacturer's label. 
Table 1. Insecticides for Use on Vegetables

\begin{tabular}{|c|c|c|c|c|}
\hline Insecticide & General Characteristics & Signal Word & MOA $^{1}$ & Typical Target Pests \\
\hline \multicolumn{5}{|l|}{ Carbamates } \\
\hline $\begin{array}{l}\text { *Lannate } \\
\text { (methomyl) }\end{array}$ & very short residual & Danger-Poison & $1 \mathrm{~A}$ & caterpillars, leafhoppers \\
\hline $\begin{array}{l}\text { Larvin } \\
\text { (thiodicarb) }\end{array}$ & larvicide \& ovicide & Warning & $1 \mathrm{~A}$ & caterpillars \\
\hline $\begin{array}{l}\text { Sevin } \\
\text { (carbaryl) }\end{array}$ & $\begin{array}{l}\text { use can result in aphid and } \\
\text { mite outbreaks }\end{array}$ & $\begin{array}{l}\text { Caution-4F, XLR, } \\
\text { Bait; Warning-80S }\end{array}$ & $1 \mathrm{~A}$ & $\begin{array}{l}\text { beetles, leafhoppers, } \\
\text { caterpillars }\end{array}$ \\
\hline $\begin{array}{l}\text { *Vydate } \\
\text { (oxamyl) }\end{array}$ & $\begin{array}{l}\text { contact action; systemic if } \\
\text { applied to soil }\end{array}$ & Danger-Poison & $1 \mathrm{~A}$ & $\begin{array}{l}\text { aphids, thrips, some } \\
\text { beetles }\end{array}$ \\
\hline \multicolumn{5}{|l|}{ Organophosphates } \\
\hline $\begin{array}{l}{ }^{*} \text { Counter } \\
\text { (terbufos) }\end{array}$ & $\begin{array}{l}\text { systemic action; } \\
\text { insecticide/nematicide }\end{array}$ & Danger-Poison & $1 \mathrm{~B}$ & soil pests \\
\hline *Diazinon & soil application & Caution & $1 \mathrm{~B}$ & $\begin{array}{l}\text { cutworms, mole crickets, } \\
\text { wireworms }\end{array}$ \\
\hline $\begin{array}{l}\text { Dibrom 8E } \\
\text { (naled) }\end{array}$ & $\begin{array}{l}\text { some short residual } \\
\text { fumigant action }\end{array}$ & Danger & 1B & caterpillars \\
\hline Dimethoate 4EC & local systemic & Warning & $1 \mathrm{~B}$ & aphids, leafhoppers \\
\hline $\begin{array}{l}\text { *Di-Syston } 8 \\
\text { (disulfoton) }\end{array}$ & systemic action & Danger-Poison & $1 \mathrm{~B}$ & aphids \\
\hline $\begin{array}{l}\text { Imidan 70W } \\
\text { (phosmet) }\end{array}$ & & Warning & $1 \mathrm{~B}$ & $\begin{array}{l}\text { caterpillars, sweetpotato } \\
\text { weevil }\end{array}$ \\
\hline $\begin{array}{l}\text { Lorsban } \\
\text { (chlorpyrifos) }\end{array}$ & long residual & $\begin{array}{l}\text { Caution-15G } \\
\text { Warning-75WG, } \\
\text { *Advanced }\end{array}$ & $1 \mathrm{~B}$ & caterpillars, soil pests \\
\hline Malathion & short residual & $\begin{array}{l}\text { Warning }-57 \mathrm{EC} \\
\text { Caution }-8 \mathrm{~F}\end{array}$ & $1 \mathrm{~B}$ & broad spectrum \\
\hline $\begin{array}{l}\text { *MSR Spray Concentrate } \\
\text { (oxydemetonmethyl) }\end{array}$ & $\begin{array}{l}\text { systemic; contact \& } \\
\text { stomach action }\end{array}$ & Danger-Poison & $1 \mathrm{~B}$ & $\begin{array}{l}\text { aphids, thrips \& other } \\
\text { sucking insects }\end{array}$ \\
\hline $\begin{array}{l}\text { *Mocap } \\
\text { (ethoprop) }\end{array}$ & contact action & Danger-Poison & $1 \mathrm{~B}$ & aphids, caterpillars \\
\hline $\begin{array}{l}\text { *Monitor } \\
\text { (methamidophos) }\end{array}$ & $\begin{array}{l}\text { insecticide/nematicide; } \\
\text { long residual }\end{array}$ & Danger-Poison & $1 \mathrm{~B}$ & caterpillars \& other pests \\
\hline $\begin{array}{l}\text { Orthene } 97 \\
\text { (acephate) }\end{array}$ & $\begin{array}{l}10-15 \text { day residual } \\
\text { systemic activity }\end{array}$ & Caution & 1B & $\begin{array}{l}\text { aphids, beetles, caterpillars, } \\
\text { thrips }\end{array}$ \\
\hline $\begin{array}{l}\text { *Penncap-M } \\
\text { (methyl parathion) }\end{array}$ & $\begin{array}{l}\text { contact \& fumigant action; } \\
\text { slow-release formulation }\end{array}$ & Warning & $1 \mathrm{~B}$ & caterpillars, thrips \\
\hline $\begin{array}{l}\text { *Thimet 20G } \\
\text { (phorate) }\end{array}$ & systemic action & Danger-Poison & $1 \mathrm{~B}$ & soil pests, thrips \\
\hline \multicolumn{5}{|l|}{ Organochlorines } \\
\hline $\begin{array}{l}\text { *Endosulfan } \\
\text { (endosulfan) }\end{array}$ & fairly long residual & Danger-Poison & $2 \mathrm{~A}$ & $\begin{array}{l}\text { aphids, beetles, caterpillars, } \\
\text { whiteflies }\end{array}$ \\
\hline \multicolumn{5}{|l|}{ Pyrethroids } \\
\hline $\begin{array}{l}{ }^{*} \text { Ambush } 25 \mathrm{~W} \\
\text { (permethrin) }\end{array}$ & $\begin{array}{l}\text { rapid knockdown; } \\
\text { repellent; broad spectrum }\end{array}$ & Warning & 3 & $\begin{array}{l}\text { beetles, caterpillars, } \\
\text { leafhoppers, thrips }\end{array}$ \\
\hline $\begin{array}{l}\text { *Asana XL } \\
\text { (esfenvalerate) }\end{array}$ & $\begin{array}{l}\text { rapid knockdown; } \\
\text { repellent; broad spectrum }\end{array}$ & Warning & 3 & $\begin{array}{l}\text { beetles, caterpillars, } \\
\text { leafhoppers }\end{array}$ \\
\hline $\begin{array}{l}\text { *Baythroid XL } \\
\text { (beta-cyfluthrin) }\end{array}$ & $\begin{array}{l}\text { rapid knockdown; } \\
\text { repellent; broad spectrum }\end{array}$ & Warning & 3 & $\begin{array}{l}\text { beetles, caterpillars, } \\
\text { leafhoppers, thrips }\end{array}$ \\
\hline $\begin{array}{l}\text { *Brigade } 2 \mathrm{EC} \\
\text { (bifenthrin) }\end{array}$ & $\begin{array}{l}\text { rapid knockdown; } \\
\text { repellent; broad spectrum }\end{array}$ & Warning & 3 & $\begin{array}{l}\text { beetles, caterpillars, } \\
\text { leafhoppers, thrips, } \\
\text { whiteflies }\end{array}$ \\
\hline
\end{tabular}




\begin{tabular}{|c|c|c|c|c|}
\hline Insecticide & General Characteristics & Signal Word & MOA $^{1}$ & Typical Target Pests \\
\hline $\begin{array}{l}\text { *Danitol 2.4 EC } \\
\text { (fenpropathrin) }\end{array}$ & $\begin{array}{l}\text { rapid knockdown; } \\
\text { repellent; broad spectrum }\end{array}$ & Warning & 3 & $\begin{array}{l}\text { caterpillars, leafhoppers, } \\
\text { whiteflies }\end{array}$ \\
\hline $\begin{array}{l}\text { *Force } \\
\text { (tefluthrin) }\end{array}$ & broad spectrum & Caution & 3 & soil pests \\
\hline *Mustang Max (zeta-cypermethrin) & $\begin{array}{l}\text { rapid knockdown, } \\
\text { repellent, broad spectrum }\end{array}$ & Warning & 3 & $\begin{array}{l}\text { beetles, caterpillars, } \\
\text { leafhoppers, thrips }\end{array}$ \\
\hline $\begin{array}{l}\text { *Pounce } \\
\text { (permethrin) }\end{array}$ & $\begin{array}{l}\text { rapid knockdown; } \\
\text { repellent; broad spectrum }\end{array}$ & $\begin{array}{l}\text { Caution-1.5G } \\
\text { Warning-25WP }\end{array}$ & 3 & $\begin{array}{l}\text { beetles, caterpillars, } \\
\text { leafhoppers, thrips }\end{array}$ \\
\hline $\begin{array}{l}\text { *Proaxis } \\
\text { (gamma-cyhalothrin) }\end{array}$ & $\begin{array}{l}\text { rapid knockdown; } \\
\text { repellent; broad spectrum }\end{array}$ & Caution & 3 & $\begin{array}{l}\text { beetles, caterpillars, } \\
\text { leafhoppers, plant bugs, } \\
\text { stink bugs }\end{array}$ \\
\hline Pyganic 5.0 (Pyrethrins) & $\begin{array}{l}\text { contact, stomach, \& } \\
\text { fumigant action; extract } \\
\text { from chrysanthemums }\end{array}$ & Caution & 3 & broad spectrum \\
\hline $\begin{array}{l}\text { *Warrior II } \\
\text { (lambda-cyhalothrin) }\end{array}$ & $\begin{array}{l}\text { rapid knockdown; } \\
\text { repellent; broad spectrum }\end{array}$ & Warning & 3 & $\begin{array}{l}\text { beetles, caterpillars, } \\
\text { leafhoppers, thrips }\end{array}$ \\
\hline \multicolumn{5}{|l|}{ Neonicotinyls } \\
\hline $\begin{array}{l}\text { Actara } \\
\text { (thiamethoxam) }\end{array}$ & local systemic & Caution & $4 \mathrm{~A}$ & $\begin{array}{l}\text { aphids, potato leafhopper, } \\
\text { some beetles, stinkbugs, } \\
\text { whiteflies }\end{array}$ \\
\hline $\begin{array}{l}\text { Admire Pro, generics } \\
\text { (imidacloprid) }\end{array}$ & $\begin{array}{l}\text { systemic or locally } \\
\text { systemic, depending on } \\
\text { application method; long } \\
\text { residual }\end{array}$ & Caution & $4 \mathrm{~A}$ & $\begin{array}{l}\text { aphids, leafhoppers, some } \\
\text { beetles, whiteflies }\end{array}$ \\
\hline $\begin{array}{l}\text { Assail 30SG } \\
\text { (acetamiprid) }\end{array}$ & $\begin{array}{l}\text { local systemic; ovicidal } \\
\text { effects }\end{array}$ & Caution & $4 \mathrm{~A}$ & $\begin{array}{l}\text { aphids, Colorado potato } \\
\text { beetle, whiteflies }\end{array}$ \\
\hline $\begin{array}{l}\text { Belay } \\
\text { (clothianidin) }\end{array}$ & systemic; long residual & Caution & $4 \mathrm{~A}$ & $\begin{array}{l}\text { Colorado potato beetle, } \\
\text { aphids, leafhoppers }\end{array}$ \\
\hline $\begin{array}{l}\text { Platinum 75SG } \\
\text { (thiamethoxam) }\end{array}$ & $\begin{array}{l}\text { systemic; soil application; } \\
\text { long residual }\end{array}$ & Caution & $4 \mathrm{~A}$ & $\begin{array}{l}\text { aphids, potato leafhopper, } \\
\text { some beetles, stinkbugs, } \\
\text { whiteflies }\end{array}$ \\
\hline $\begin{array}{l}\text { Venom } \\
\text { Scorpion } \\
\text { (dinotefuran) }\end{array}$ & $\begin{array}{l}\text { systemic or locally } \\
\text { systemic, depending on } \\
\text { application method; long } \\
\text { residual }\end{array}$ & Caution & $4 \mathrm{~A}$ & $\begin{array}{l}\text { aphids, Colorado potato } \\
\text { beetle, leafhoppers, } \\
\text { leafminers, thrips, } \\
\text { whiteflies }\end{array}$ \\
\hline \multicolumn{5}{|l|}{ Other insect nerve poisons } \\
\hline $\begin{array}{l}{ }^{*} \text { Agri-Mek SC } \\
\text { (abamectin) }\end{array}$ & $\begin{array}{l}\text { active once ingested; some } \\
\text { contact action; mostly } \\
\text { stomach poison }\end{array}$ & Warning & 6 & $\begin{array}{l}\text { leafminers, mites, some } \\
\text { beetles, tomato pinworm }\end{array}$ \\
\hline $\begin{array}{l}\text { Avaunt } \\
\text { (indoxacarb) }\end{array}$ & $\begin{array}{l}\text { ingestion plus contact; } \\
\text { slightly to moderately } \\
\text { translaminar }\end{array}$ & Caution & 22 & caterpillars \\
\hline $\begin{array}{l}\text { Beleaf 50SG } \\
\text { (flonicamid) }\end{array}$ & $\begin{array}{l}\text { contact \& ingestion; causes } \\
\text { rapid cessation of feeding }\end{array}$ & Caution & $9 \mathrm{C}$ & aphids \\
\hline $\begin{array}{l}\text { Coragen } \\
\text { (chlorantraniliprole) }\end{array}$ & $\begin{array}{l}\text { long residual, especially } \\
\text { when applied to soil; } \\
\text { systemic }\end{array}$ & None & 28 & $\begin{array}{l}\text { caterpillars, Colorado } \\
\text { potato beetle }\end{array}$ \\
\hline $\begin{array}{l}\text { Fulfill } \\
\text { (pymetrozine) }\end{array}$ & feeding inhibitor & Caution & $9 \mathrm{~B}$ & aphids, whiteflies \\
\hline $\begin{array}{l}\text { *Proclaim } \\
\text { (emamectin benzoate) }\end{array}$ & $\begin{array}{l}\text { ingestion \& topical; } \\
\text { translaminar, not systemic }\end{array}$ & Caution & 6 & caterpillars \\
\hline $\begin{array}{l}\text { Radiant } \\
\text { (spinetoram) }\end{array}$ & $\begin{array}{l}\text { ingestion \& contact; } \\
\text { enters leaf but does not } \\
\text { translocate }\end{array}$ & Caution & 5 & $\begin{array}{l}\text { thrips, caterpillars, some } \\
\text { beetles and leafminers }\end{array}$ \\
\hline
\end{tabular}




\begin{tabular}{|c|c|c|c|c|}
\hline Insecticide & General Characteristics & Signal Word & MOA $^{1}$ & Typical Target Pests \\
\hline $\begin{array}{l}\text { SpinTor, Entrust } \\
\text { (spinosad) }\end{array}$ & $\begin{array}{l}\text { ingestion \& contact; } \\
\text { enters leaf but does not } \\
\text { translocate }\end{array}$ & Caution & 5 & $\begin{array}{l}\text { thrips caterpillars, some } \\
\text { beetles and leafminers }\end{array}$ \\
\hline $\begin{array}{l}\text { Synapse WG, Belt } \\
\text { (flubendiamide) }\end{array}$ & $\begin{array}{l}\text { long residual; rapid } \\
\text { cessation of feeding }\end{array}$ & Caution & 28 & caterpillars \\
\hline \multicolumn{5}{|l|}{ Insect Growth Regulators } \\
\hline $\begin{array}{l}\text { Confirm 2F } \\
\text { (tebufenozide) }\end{array}$ & $\begin{array}{l}\text { slow-acting; } \\
\text { safe for beneficials }\end{array}$ & Caution & 18 & caterpillars \\
\hline $\begin{array}{l}\text { Courier } 40 S C \\
\text { (buprofezin) }\end{array}$ & $\begin{array}{l}\text { disrupts egg hatch and } \\
\text { molting; use in rotation } \\
\text { with other insecticides }\end{array}$ & Caution & 16 & whiteflies \\
\hline $\begin{array}{l}\text { *Dimilin } \\
\text { (diflubenzuron) }\end{array}$ & $\begin{array}{l}\text { slow-acting; disrupts } \\
\text { molting process; reduces } \\
\text { egg hatch }\end{array}$ & Caution & 15 & caterpillars, pepper weevil \\
\hline $\begin{array}{l}\text { Esteem Ant Bait } \\
\text { (pyriproxyfen) }\end{array}$ & $\begin{array}{l}\text { bait-breaks reproductive } \\
\text { cycle of ants; slow-acting } \\
\text { but effective }\end{array}$ & Caution & 7C & ants \\
\hline $\begin{array}{l}\text { Extinguish } \\
{[(\mathrm{S}) \text {-methoprene] }}\end{array}$ & slow-acting bait & Caution & $7 \mathrm{~A}$ & fire ants \\
\hline $\begin{array}{l}\text { Intrepid } \\
\text { (methoxyfenozide) }\end{array}$ & $\begin{array}{l}\text { stomach poison that acts } \\
\text { as an IGR for Lepidoptera; } \\
\text { slow-acting; safe for } \\
\text { beneficials }\end{array}$ & Caution & 18 & caterpillars \\
\hline $\begin{array}{l}\text { Knack } \\
\text { (pyriproxyfen) }\end{array}$ & $\begin{array}{l}\text { disrupts egg hatch and } \\
\text { molting }\end{array}$ & Caution & $7 C$ & whiteflies \\
\hline $\begin{array}{l}\text { Neemix } 4.5 \text {, others } \\
\text { (azadirachtin) }\end{array}$ & $\begin{array}{l}\text { slow acting; also acts as } \\
\text { feeding repellent }\end{array}$ & $\begin{array}{l}\text { Caution-Azatin XL; } \\
\text { Warning-Neemix } \\
4.5\end{array}$ & un & broad spectrum \\
\hline $\begin{array}{l}\text { Rimon 0.83EC } \\
\text { (novaluron) }\end{array}$ & $\begin{array}{l}\text { disrupts cuticle formation } \\
\text { and deposition at molting, } \\
\text { resulting in death of larva; } \\
\text { no effect on adult insect }\end{array}$ & Warning & 15 & caterpillars \\
\hline $\begin{array}{l}\text { Trigard } \\
\text { (cyromazine) }\end{array}$ & $\begin{array}{l}\text { most effective against } \\
\text { small leafminer larvae }\end{array}$ & Caution & 17 & $\begin{array}{l}\text { dipterous leafminers, } \\
\text { maggots, Colorado potato } \\
\text { beetle }\end{array}$ \\
\hline $\begin{array}{l}\text { Zeal } \\
\text { (etoxazole) }\end{array}$ & mite growth inhibitor & Caution & $10 \mathrm{~B}$ & $\begin{array}{l}\text { spider mites on melons, } \\
\text { cucumbers }\end{array}$ \\
\hline \multicolumn{5}{|l|}{ Miscellaneous } \\
\hline $\begin{array}{l}\text { Acramite } \\
\text { (bifenazate) }\end{array}$ & $\begin{array}{l}\text { contact; long residual; } \\
\text { ovicidal activity against } \\
\text { spider mites }\end{array}$ & Caution & un & mites \\
\hline $\begin{array}{l}\text { Bacillus thuringiensis } \\
\text { (B.t.) var. aizawai } \\
\text { (B.t.) var. kurstaki }\end{array}$ & $\begin{array}{l}\text { pest must ingest; slow- } \\
\text { acting but feeding stops } \\
\text { long before death }\end{array}$ & Caution & $11 \mathrm{~A}$ & caterpillars \\
\hline Cryolite & $\begin{array}{l}\text { pest must ingest; not } \\
\text { rainfast; an inorganic } \\
\text { fluorine compound }\end{array}$ & Caution & un & beetles, caterpillars \\
\hline $\begin{array}{l}\text { Kanemite 15SC } \\
\text { (acequinocyl) }\end{array}$ & $\begin{array}{l}\text { knockdown and residual } \\
\text { control }\end{array}$ & Caution & $20 B$ & mites \\
\hline $\begin{array}{l}\text { M-Pede, Des-X } \\
\text { (potassium salts of fatty acids) }\end{array}$ & $\begin{array}{l}\text { contact activity; phytotoxic } \\
\text { at high temperatures }\end{array}$ & Warning & & $\begin{array}{l}\text { Aphids, whiteflies, } \\
\text { and other soft-bodied } \\
\text { arthropods }\end{array}$ \\
\hline
\end{tabular}




\begin{tabular}{|c|c|c|c|c|}
\hline Insecticide & General Characteristics & Signal Word & MOA $^{1}$ & Typical Target Pests \\
\hline $\begin{array}{l}\text { Movento } \\
\text { (spirotetramat) }\end{array}$ & $\begin{array}{l}\text { ingestion; fully systemic } \\
\text { in plant after foliar } \\
\text { application; inhibitor } \\
\text { of lipid synthesis; most } \\
\text { effective on juvenile stages }\end{array}$ & Caution & 23 & $\begin{array}{l}\text { aphids, psyllids, whiteflies, } \\
\text { mites }\end{array}$ \\
\hline $\begin{array}{l}\text { Mycotrol, BotaniGard } \\
\text { (Beauveria) }\end{array}$ & $\begin{array}{l}\text { entomopathogenic } \\
\text { fungus; slow-acting }\end{array}$ & None & - & $\begin{array}{l}\text { aphids, leafhoppers, } \\
\text { whiteflies }\end{array}$ \\
\hline $\begin{array}{l}\text { Oberon 2SC } \\
\text { (spiromesifen) }\end{array}$ & $\begin{array}{l}\text { inhibitor of lipid synthesis; } \\
\text { most effective on juvenile } \\
\text { stages of mites and on } \\
\text { nymphs and pupae of } \\
\text { whiteflies and psyllids }\end{array}$ & Caution & 23 & mites, psyllids, whiteflies \\
\hline $\begin{array}{l}\text { Portal } \\
\text { (fenpyroximate) }\end{array}$ & $\begin{array}{l}\text { contact activity; affects } \\
\text { energy metabolism }\end{array}$ & Warning & $21 \mathrm{~A}$ & $\begin{array}{l}\text { mites, including broad } \\
\text { mites, leafhoppers, } \\
\text { whiteflies }\end{array}$ \\
\hline $\begin{array}{l}\text { Requiem } 25 \mathrm{SC} \\
\text { (synthetic extract of Chenopodium } \\
\text { ambrosioides) }\end{array}$ & $\begin{array}{l}\text { affects insect cuticle, } \\
\text { disrupting respiration }\end{array}$ & Caution & Un & aphids, whiteflies, thrips \\
\hline $\begin{array}{l}\text { SunSpray Ultra Fine Spray Oil, others } \\
\text { (mineral oil) }\end{array}$ & contact activity & Caution & - & aphids, mites, whiteflies \\
\hline *Vendex (fenbutatin-oxide) & affects energy metabolism & Danger-Poison & $12 B$ & Mites \\
\hline \multicolumn{5}{|c|}{$\begin{array}{l}\text { Premixes (note: premixes or tank mixes reduce potential rotation partners for resistance management. Use only if both products in mix are } \\
\text { needed to control damaging pests.) }\end{array}$} \\
\hline $\begin{array}{l}\text { *Brigadier } \\
\text { (bifenthrin and imidacloprid) }\end{array}$ & see Brigade and Admire & Warning & $3 A+c 4 A$ & broad spectrum \\
\hline $\begin{array}{l}\text { *Cobalt } \\
\text { (chlorpyrifos and gamma-cyhalothrin) }\end{array}$ & see Lorsban and Proaxis & Danger & $1 B+3 A$ & broad spectrum \\
\hline $\begin{array}{l}\text { Durivo SC } \\
\text { (chlorantraniliprole and } \\
\text { thiamethoxam) }\end{array}$ & $\begin{array}{l}\text { see Platinum and Coragen; } \\
\text { applied to soil }\end{array}$ & $\begin{array}{l}\text { Keep out of reach of } \\
\text { children }\end{array}$ & $28+4 A$ & $\begin{array}{l}\text { caterpillars, aphids, potato } \\
\text { leafhopper, some beetles, } \\
\text { stinkbugs, whiteflies }\end{array}$ \\
\hline $\begin{array}{l}\text { *Endigo ZC } \\
\text { (lambda-cyhalothrin and } \\
\text { thiamethoxam) }\end{array}$ & see Warrior and Actara & Warning & $3 A+4 A$ & broad spectrum \\
\hline $\begin{array}{l}\text { *Leverage } 360 \\
\text { (beta-cyfluthrin and imidacloprid) }\end{array}$ & $\begin{array}{l}\text { see Baythroid XL and } \\
\text { Admire }\end{array}$ & Caution & $3 A+4 A$ & broad spectrum \\
\hline $\begin{array}{l}\text { Vetica } \\
\text { (buprofezin and flubendiamide) }\end{array}$ & $\begin{array}{l}\text { see Courier and Synapse } \\
\text { WG }\end{array}$ & Caution & $16+28$ & whiteflies and caterpillars \\
\hline $\begin{array}{l}\text { Voliam Flexi } \\
\text { (chlorantraniliprole and } \\
\text { thiamethoxam) }\end{array}$ & $\begin{array}{l}\text { see Coragen and Actara; } \\
\text { foliar application }\end{array}$ & Caution & $28+4 A$ & $\begin{array}{l}\text { caterpillars, aphids, potato } \\
\text { leafhopper, some beetles, } \\
\text { stinkbugs, whiteflies }\end{array}$ \\
\hline $\begin{array}{l}\text { *Voliam Xpress } \\
\text { (chlorantraniliprole and lambda- } \\
\text { cyhalothrin) }\end{array}$ & see Coragen and Warrior & Warning & $28+3 A$ & broad spectrum \\
\hline
\end{tabular}


*Restricted Use Pesticide

Originally adapted from: Welty, Celeste. Insecticides for use on vegetables in Ohio. pp. 46-48, 2002 Ohio Vegetable Production Guide, Ohio State University.

'Mode of Action codes for vegetable pest insecticides from the Insecticide Resistance Action Committee (IRAC) Mode of Action

Classification v.7.2 February 2012. http://www.irac-online.org/wp-content/uploads/MoA-classification.pdf

$1 \mathrm{~A}$. Acetylcholinesterase inhibitors, Carbamates (nerve action)

1B. Acetylcholinesterase inhibitors, Organophosphates (nerve action)

2A. GABA-gated chloride channel antagonists (nerve action)

$3 A$. Sodium channel modulators-pyrethroids

4A. Nicotinic acetylcholine receptor agonists (nerve action)

5. Nicotinic acetylcholine receptor allosteric activators-spinosins (nerve action)

6. Chloride channel activators (nerve and muscle action)

7A. Juvenile hormone mimics (growth regulation)

7C. Juvenile hormone mimics (growth regulation)

$9 B$ \& 9 C. Selective homopteran feeding blockers

10B. Mite growth inhibitors (growth regulation)

11A. Microbial disruptors of insect midgut membranes

12B. Inhibitors of mitochondrial ATP synthase (energy metabolism)

15. Inhibitors of chitin biosynthesis, type 0 , lepidopteran (growth regulation)

16. Inhibitors of chitin biosynthesis, type 1, homopteran (growth regulation)

17. Molting disruptor, dipteran (growth regulation)

18. Ecdysone receptor agonists (growth regulation)

20B. Mitochondrial complex III electron transport inhibitors (energy metabolism)

21A. Mitochondrial complex I electron transport inhibitors (energy metabolism)

22. Voltage-dependent sodium channel blockers (nerve action)

23. Inhibitors of acetyl Co-A carboxylase (lipid synthesis, growth regulation)

28. Ryanodine receptor modulators (nerve and muscle action)

un. Compounds of unknown or uncertain mode of action 